\title{
Resíduo da polpa de goiaba em dietas para codornas
}

Lana, S.R.V. ${ }^{1}$; Silva, L.C.L. ${ }^{2}$; Lana, G.R.Q. ${ }^{\text {; }}$ Leão, A.P.A. ${ }^{3}$; Barros Jr., R.F. ${ }^{4}$; Santos, T.M.C. ${ }^{1}$ e Santos, D.S. ${ }^{1}$

'Departamento de Zootecnia. Universidade Federal de Alagoas. Maceió. Alagoas. Brasil.

${ }^{2}$ Mestre em Zootecnista. Maceió. Alagoas. Brasil.

${ }^{3}$ Departamento de Zootecnia. Universidade Federal de Lavras. Lavras, Minas Gerais. Brasil.

${ }^{4}$ Instituto Federal de Alagoas. Satuba, Alagoas. Brasil.

\section{PALAVRAS CHAVE ADICIONAIS}

Coturnicultura.

Psidium guajava L..

Subproduto da agroindústria.

\section{RESUMO}

Objetivou-se avaliar a inclusão do resíduo de polpa de goiaba em dietas de codornas de corte sobre o desempenho produtivo, rendimento de carcaça, e viabilidade econômica, aos 42 dias de idade. Foram utilizadas 250 codornas europeias, não sexadas, de um dia de idade, distribuídas em delineamento experimental inteiramente casualizado com cinco níveis de inclusão $(0,0 ; 3,0 ; 6,0 ; 9,0$ e $12,0 \%$ ) de resíduo de goiaba, com cinco repetições e 10 aves por unidade experimental. As variáveis avaliadas durante o período experimental foram: consumo de ração (CR), ganho de peso (GP) e conversão alimentar $(C A)$, rendimento de carcaça, de cortes e visceras. Não foram observadas diferenças significativas $(P>0,05)$ para o ganho de peso, o consumo de ração e conversão alimentar das aves durante o período de um a 42 dias de idade. Os pesos absolutos e os rendimentos de carcaça, cortes nobres (peito, coxa e sobrecoxa), vísceras comestíveis e gordura abdominal das aves não foram influenciados $(\mathrm{P}>0,05)$ pelos níveis de inclusão do resíduo de polpa de goiaba às dietas. $O$ resíduo de polpa de goiaba pode ser utilizado como ingrediente alternativo nas dietas de codornas europeias, até o nível de $3 \%$ de inclusão, sem comprometer o desempenho produtivo, o rendimento de carcaça das aves e a viabilidade econômica.

\section{Residue of guava pulp in quails diets}

\section{SUMMARY}

\author{
ADDITIONAL KEYWORDS \\ Byproduction of agroindustry. \\ Psidium guajava L. \\ Quail production \\ INFORMATION \\ Cronología del artíiculo. \\ Recibido/Received: 28.08 .2018 \\ Aceptado/Accepted: 05.03.2020 \\ On-line: 15.04 .2020 \\ Correspondencia a los autores/Contact e-mail: \\ sandraroselilana@gmail.com
}

\begin{abstract}
The objective of this study was to evaluate the inclusion of the guava pulp residue in broiler quail diets on productive performance, carcass yield, and poultry economic viability at 42 days of age. Two hundred and fifty-one unsexed European quail were distributed in a completely randomized experimental design with five inclusion levels $(0.0,3.0,6.0,9.0$ and $12.0 \%)$ of residue of guava, with five replicates and 10 birds per experimental unit. The variables evaluated during the experimental period were: feed intake (FI), weight gain (WG) and feed conversion (FC), carcass yield, noble cuts and edible offal. No significant differences $(P>0.05)$ were observed for weight gain, feed intake and feed conversion rate during 1 to 42 days of age. The absolute weights and carcass yields, noble cuts (breast, thigh and drumstick), edible offal and abdominal fat of the quails were not influenced $(P>0.05)$ by the inclusion levels of the guava pulp residue to the diets. The guava pulp residue can be used as an alternative ingredient in European quail diets up to $3 \%$ inclusion level without compromising yield performance, poultry carcass yield and economic viability.
\end{abstract}

\section{INTRODUÇÃO}

A avicultura tem evoluído muito nos últimos anos, se tornando um segmento de grande importância na produção de alimentos de alto valor proteico, buscado se adequar a novas técnicas que proporcionam melhoria na eficiência produtiva das aves. Esses fatores fizeram com que o Brasil recebesse destaque no ranking mundial de produção e exportação de aves, ocupando o segundo lugar e primeiro lugar respectivamente (ABPA,2018).

A coturnicultura apresenta-se como o segmento avícola de maior crescimento, inserindo-se cada vez mais no contexto de produção industrial de corte e postura. Entretanto, a maioria das rações produzidas para monogástricos é formulada à base de milho e farelo de soja, que são ingredientes de custo elevado, por isso há um crescente interesse por alimentos alternativos que possam ser utilizados em dietas desses animais, sem prejuízo ao seu desempenho, visando tornar as rações mais econômicas, pela possibilidade de substituição do milho e do farelo de soja (Tardocchi et al., 2014).

O Brasil é um dos maiores produtores mundiais de frutas, com uma produção de 424,305 toneladas no ano de 2016. Com essa alta produção a fruticultura 
é responsável por uma produção enorme de resíduos provenientes do processamento das frutas (ABF, 2017). Entre os diversos alimentos alternativos destacam-se os resíduos das agroindústrias de doce, sucos e extratos vegetais que geram uma grande quantidade de subprodutos oriundos do tratamento industrial, tais como: sementes, polpas e cascas, potenciais fontes substitutivas parciais em dietas de monogástricos, diminuindo, assim, a inclusão de milho e farelo de soja nas rações (Lira et al., 2009). No entanto, esses resíduos podem apresentar valor nutricional variável, podendo ser de baixa qualidade, por influência da forma de processamento e das substâncias utilizadas neste processamento, o que pode limitar a quantidade a ser incluída nas rações. Portanto, são necessários estudos para avaliar o valor nutricional desses subprodutos, visando a sua inclusão racional na alimentação de monogástricos, uma vez que, se forem fornecidos de forma exclusiva, podem não atender às necessidades dos animais (Lira et al., 2011).

Dentre os diferentes resíduos agroindustriais o resíduo de goiaba (Psidium guajava L.) se destaca, por apresentar em sua composição de vitaminas, minerais, fibras e compostos antioxidantes importantes para as funções fisiológicas, fazendo com que esse resíduo tenha uma melhor perspectiva de utilização do ponto de vista nutricional (Sousa et. al., 2011).

Com relação à composição química do resíduo da goiaba, este apresenta valores de matéria seca de $47,04 \%$ a 50,38\% (Santos et al., 2009 e Lira et al. 2009) e valores variáveis de proteína bruta de 8,6 a 10,90\% (Silva, 1999; Santos et al., 2009 e Lira et al. 2009); de fibra bruta de 43,44 a 61,25\% (Sales et al., 2004; Silva et al., 2009 e Lira et al., 2009); de fibra em detergente neutro de 48,81 a $81,95 \%$ (Sales et al., 2004; Silva et al., 2009 e Lira et al., 2009). Embora a literatura seja escassa, quanto a utilização do resíduo de polpa de goiaba na alimentação de aves, estudos realizados por Lira et al. (2009) avaliando a inclusão de níveis de resíduo de goiaba às dietas de frangos de corte, permitiram concluir que a inclusão do resíduo em até $12 \%$ não comprometeu o desempenho produtivo dos frangos aos 42 dias de idade. Da mesma forma, Camelo et al. (2015) recomendaram a inclusão de até $10 \%$ de resíduo de goiaba nas rações de codornas de oito aos 38 dias de idade.

Dentro deste contexto, objetivou-se avaliar a inclusão do resíduo da polpa de goiaba na alimentação de codornas de corte sobre o desempenho produtivo, rendimento de carcaça e a viabilidade econômica, durante o período de um a 42 dias de idade.

\section{MATERIAL E MÉTODOS}

Todos os procedimentos deste estudo foram aprovados pelo Comitê de Ética no Uso de Animais da UFAL, sob número de protocolo nº 86/2016. Dois experimentos foram realizados no setor de Coturnicultura do Centro de Ciências Agrárias da Universidade Federal de Alagoas - UFAL, localizado no município de Rio Largo - AL.
Foram utilizadas 250 codornas, não sexadas, com um dia de idade, da espécie europeia (Coturnix Coturnix) provenientes da Granja Fujikura, localizada no Estado de São Paulo. As aves foram selecionadas de acordo com o peso médio inicial de 8,23g $\pm 0,20$ e alojadas em gaiolas do tipo bateria, com $50 \mathrm{~cm}$ de comprimento, $60 \mathrm{~cm}$ de largura e $30 \mathrm{~cm}$ de altura, em galpão com piso de concreto durante o período de um a 42 dias de idade.

A alimentação foi fornecida em comedouros e bebedouros próprios para a fase de criação; a ração e a água foram fornecidas ad libitum durante todo o período experimental. $\mathrm{O}$ delineamento adotado foi inteiramente ao acaso, com cinco tratamentos $(0,00$; 3,$0 ; 6,0 ; 9,0$ e $12,0 \%$ de inclusão do resíduo de polpa de goiaba), cinco repetições e 10 aves por unidade experimental.

As rações experimentais foram formuladas à base de milho e farelo de soja, visando atender às exigências nutricionais das aves, segundo recomendações preconizadas por Rostagno et al. (2011), sendo isocalóricas e isonutritivas (Tabela I). O resíduo da goiaba, composto por sementes, cascas e polpa, foi oriundo do processamento agroindustrial da goiaba, fornecido por uma indústria de sucos local. O processamento foi feito por meio de secagem ao sol por oito horas, durante 15 dias, e o resíduo foi moído em forma de farelo para a inclusão na ração das aves.

A composição química e o valor energético do resíduo foram determinados utilizando a metodologia descrita por Silva \& Queiroz (2002). O resíduo da polpa de goiaba continha $13,30 \%$ de proteína bruta; $12,92 \%$ de extrato etéreo; $68,37 \%$ de fibra em detergente neutro; $52,20 \%$ de fibra em detergente ácido; $21,10 \%$ de lignina; 5.275,46 kcal kg-1 de energia bruta e 1.365

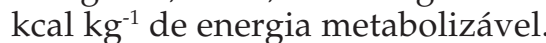

O monitoramento da temperatura e umidade relativa do ar foi realizado por meio de termohigrômetro digital, situado na altura das gaiolas, realizando-se as leituras diariamente nos horários de 08:00 e 16:00 horas. O programa de luz adotado foi o contínuo, 24 horas de luz natural+artificial, sendo esta última, utilizando lâmpadas incandescentes de 40 W. As médias das variáveis foram: temperatura máxima 27,66 ${ }^{\circ} \mathrm{C}$, temperatura mínima $26,90{ }^{\circ} \mathrm{C}$, umidade relativa $81,63 \%$ e o índice de temperatura de globo negro e umidade 72,55 , calculado de acordo com a fórmula proposta por Buffington et al. (1981).

As variáveis avaliadas durante o período experimental foram: consumo de ração (CR), ganho de peso (GP) e conversão alimentar (CA), rendimento de carcaça, de cortes e visceras. As aves, a ração fornecida e as sobras de ração foram pesadas semanalmente para o cálculo dos índices de desempenho.

Aos 42 dias de idade, as aves foram pesadas após jejum alimentar de seis horas, sendo selecionadas duas aves por unidade experimental com peso vivo próximo da média de peso observado para a referida gaiola, para posterior realização dos procedimentos normais de abate (atordoamento, sangria e depenagem), conforme preconizados pelo Regulamento de 


\begin{tabular}{|c|c|c|c|c|c|}
\hline \multirow[t]{2}{*}{ Ingredientes (\%) } & \multicolumn{5}{|c|}{ Níveis de inclusão do resíduo da goiaba (\%) } \\
\hline & 0,0 & 3,0 & 6,0 & 9,0 & 12,0 \\
\hline Milho & 57,022 & 52,929 & 48,837 & 44,744 & 40,651 \\
\hline Farelo de Soja (45\%) & 38,434 & 38,372 & 38,309 & 38,247 & 38,184 \\
\hline Resíduo da goiaba & 0,000 & 3,000 & 6,000 & 9,000 & 12,00 \\
\hline Fosfato bicálcico & 1,385 & 1,399 & 1,413 & 1,427 & 1,441 \\
\hline Calcário & 1,164 & 1,159 & 1,153 & 1,148 & 1,142 \\
\hline Sal Comum & 0,395 & 0,397 & 0,399 & 0,401 & 0,403 \\
\hline Óleo de Soja & 1,109 & 2,222 & 3,226 & 4,450 & 5,564 \\
\hline DL-Metionina & 0,125 & 0,132 & 0,138 & 0,145 & 0,152 \\
\hline L-Lisina $\mathrm{HCl}$ & 0,030 & 0,042 & 0,054 & 0,066 & 0,078 \\
\hline L-Treonina & 0,033 & 0,045 & 0,057 & 0,069 & 0,082 \\
\hline Suplemento Vitamínico ${ }^{1}$ & 0,050 & 0,050 & 0,050 & 0,050 & 0,050 \\
\hline Suplemento Mineral ${ }^{2}$ & 0,100 & 0,100 & 0,100 & 0,100 & 0,100 \\
\hline Bac-zinco & 0,050 & 0,050 & 0,050 & 0,050 & 0,050 \\
\hline Cygro & 0,050 & 0,050 & 0,050 & 0,050 & 0,050 \\
\hline BHT & 0,050 & 0,050 & 0,050 & 0,050 & 0,050 \\
\hline Inerte & 0,000 & 0,000 & 0,000 & 0,000 & 0,000 \\
\hline Total & 100,00 & 100,00 & 100,00 & 100,00 & 100,00 \\
\hline \multicolumn{6}{|l|}{ Composição calculada } \\
\hline Energia Met. Aves (Kcal/kg) & 2.9000 & 2.9000 & 2.9000 & 2.9000 & 2.9000 \\
\hline Proteína Bruta (\%) & 22,00 & 22,00 & 22,00 & 22,00 & 22,00 \\
\hline Cálcio total (\%) & 0,900 & 0,900 & 0,900 & 0,900 & 0,900 \\
\hline Fósforo Disponível (\%) & 0,375 & 0,375 & 0,375 & 0,375 & 0,375 \\
\hline Sódio (\%) & 0,176 & 0,176 & 0,176 & 0,176 & 0,176 \\
\hline Metionina Total (\%) & 0,460 & 0,460 & 0,460 & 0,460 & 0,460 \\
\hline Met. Digestível (\%) & 0,420 & 0,420 & 0,420 & 0,420 & 0,420 \\
\hline Met.+cistina total (\%) & 0,844 & 0,844 & 0,844 & 0,844 & 0,844 \\
\hline Lisina Total (\%) & 1,244 & 1,244 & 1,244 & 1,244 & 1,244 \\
\hline Lisina Digestível (\%) & 1,120 & 1,120 & 1,120 & 1,120 & 1,120 \\
\hline Treonina Total (\%) & 0,915 & 0,915 & 0,915 & 0,915 & 0,915 \\
\hline Treonina Digestível (\%) & 0,790 & 0,790 & 0,790 & 0,790 & 0,790 \\
\hline
\end{tabular}

Suplemento Vitamínico/kg: Vit.A 13.440,000 UI; Vit. D 3.200,000 UI Vit.E 28.000 mg/kg; Vit.K 2.880 mg/kg; Tiamina 3.500 mg/kg; Riboflavina $9.600 \mathrm{mg} / \mathrm{kg}$; Piridoxina $5.000 \mathrm{mg} / \mathrm{kg}$; Cianocobalamina 19.200 mcg/kg; Ácido Fólico $1.600 \mathrm{mg} / \mathrm{kg}$; Ácido Pantotênico 25,000 $\mathrm{mg} / \mathrm{kg}$; Niacina $67.200 \mathrm{mg} / \mathrm{kg}$; Biotina $80.000 \mathrm{mcg} / \mathrm{kg}$; Selênio 600 ppm; Antioxidante 0,40 g/kg.

2Suplemento Mineral/kg: Mg 150.000 ppm; Zn 140.000 ppm; Fe 100.000 ppm; Cu 16.000 ppm; I 1.500 ppm.

Tabela II. Consumo de ração (CR), ganho de peso (GP) e conversão alimentar (CA) de codornas de corte alimentadas com diferentes níveis de inclusão do resíduo de goiaba na dieta (Feed intake (FI), weight gain (WG) and feed conversion (FC) of broiler quail fed with different inclusion levels of guava residue in the diet).

\begin{tabular}{|c|c|c|c|c|c|c|c|}
\hline \multirow[b]{2}{*}{ Variáveis } & \multicolumn{7}{|c|}{ Níveis de Inclusão do resíduo da goiaba $(\%)^{\text {NS }}$} \\
\hline & 0,0 & 3,0 & 6,0 & 9,0 & 12,0 & P-valor & EPN \\
\hline & \multicolumn{7}{|c|}{01 a 21 dias de idade } \\
\hline CR & 252,80 & 251,20 & 266,00 & 260,80 & 263,00 & 0,51 & 3,11 \\
\hline GP & 127,90 & 130,20 & 132,50 & 129,70 & 133,50 & 0,66 & 1,26 \\
\hline CA & 1,97 & 1,93 & 2,01 & 2,01 & 1,96 & 0,46 & 0,01 \\
\hline \multicolumn{8}{|c|}{22 a 42 dias de idade } \\
\hline CR & 606,10 & 643,70 & 627,80 & 641,30 & 643,80 & 0,31 & 6,58 \\
\hline GP & 121,70 & 132,00 & 128,80 & 131,10 & 138,40 & 0,44 & 2,72 \\
\hline $\mathrm{CA}$ & 5,03 & 4,89 & 4,89 & 4,95 & 4,66 & 0,76 & 0,08 \\
\hline \multicolumn{8}{|c|}{01 a 42 dias de idade } \\
\hline CR & 858,80 & 894,90 & 893,80 & 902,10 & 906,80 & 0,41 & 8,31 \\
\hline GP & 249,60 & 262,20 & 261,30 & 260,70 & 271,90 & 0,22 & 2,96 \\
\hline CA & 3,44 & 3,41 & 3,42 & 3,46 & 3,33 & 0,66 & 0,02 \\
\hline
\end{tabular}


Inspeção Industrial e Sanitária dos Produtos de Origem A̧nimal (Brasil, 1997). As aves foram evisceradas, as carcaças e as vísceras foram pesadas, para posteriormente proceder-se à avaliação do rendimento de carcaça. Os parâmetros avaliados foram: pesos absolutos (g) e relativos (\%) da carcaça, de cortes nobres (peito e pernas) e das vísceras comestíveis (coração, fígado e moela).

A análise estatística foi realizada utilizando o software R Core Team (R Development..., 2016), Os dados de desempenho e de características de carcaça foram submetidos à análise de variância, utilizando o PROC ANOVA. Todos os dados foram submetidos a análise de regressão para determinar o melhor nível de inclusão do resíduo de polpa de goiaba a 5\% de probabilidade. A análise econômica foi realizada de acordo com a metodologia descrita por Lana, (2000).

A avaliação econômica das rações experimentais foi baseada nos dados de desempenho das aves. $\mathrm{O}$ preço codorna viva $\mathrm{kg}^{-1}$ e os valores das matériasprimas utilizadas para o cálculo dos custos das rações, referem-se aos valores vigentes no comércio local em maio de 2017.

\section{RESULTADOS E DISCUSSÃO}

Na Tabela II, são apresentados os resultados referentes ao consumo de ração (CR), ganho de peso (GP) e conversão alimentar (CA) de codornas de corte nos períodos de um a 21, 22 a 42 e um a 42 dias de idade submetido a níveis de inclusão do resíduo de goiaba na dieta. Não houve influência $(P>0,05)$ dos níveis de inclusão do resíduo da polpa de goiaba sobre o consumo de ração, o ganho de peso e a conversão alimentar das aves nos períodos de um a 21; 22 a 42 e um a 42 dias de idade. Este resultado indica que a utilização do resíduo da polpa de goiaba não comprometeu o desempenho produtivo das codornas.

Apesar de não ter ocorrido efeito $(\mathrm{P}>0,05)$ dos níveis de inclusão de resíduo de goiaba, pode-se observar, em valores absolutos, aumento de $5,29 \%$ no consumo de ração, de $8,20 \%$ no ganho de peso e melhora de $3,19 \%$ na conversão alimentar das aves aos 42 dias de idade. $\mathrm{O}$ fato de o resíduo de goiaba possuir alto teor de fibra em detergente neutro, fibra em detergente ácido e lignina, provavelmente, tenha contribuído para essa resposta, visto que a elevada quantidade de fibras é um fator diluidor de outros nutrientes, atua como barreira física, impedindo que as enzimas endógenas tenham acesso ao conteúdo interno das células vegetais, causando a redução do processo digestivo e, consequentemente, a absorção dos nutrientes (Jansen \& Carré, 1989), acarretando em aumento no consumo de ração das aves, a fim de compensar o aporte energético. Tal fato foi justificado por Murakami et al. (1993) ao afirmarem que, para codornas, é possível aplicar a teoria quimiostática, ou seja, a de que os animais regulam seu consumo de acordo com a necessidade de energia, no entanto, outros fatores também podem influenciar o consumo, como a necessidade da ave, o peso corporal, a fase produtiva, o crescimento, a mantença e o ambiente de criação.
Os resultados obtidos no presente estudo corroboraram os constatados por Lira et al. (2009) e Camelo et al. (2015) ao avaliarem os níveis de inclusão do resíduo de goiaba na alimentação de frangos de corte e de codornas, respectivamente. Da mesma forma, Zanetti et al. (2014) e Ramos et al. (2006) não encontraram efeitos significativos sobre o desempenho produtivo ao avaliarem, respectivamente, a inclusão do resíduo da acerola e de resíduo de polpa de caju desidratada na alimentação de frangos de corte. Por outro lado, Lira et al. (2010) e Pinto et al. (2014) verificaram influência no desempenho produtivo de frangos de corte alimentados com dietas contendo resíduo de tomate e o coproduto da acerola, respectivamente.

Vale ressaltar que, em razão de o resíduo de polpa de goiaba ser um ingrediente que contém cerca de $1.358 \mathrm{kcal} \mathrm{kg}^{-1}$ de EM, fez-se necessária a adição de óleo vegetal para suprir as exigências energéticas das codornas, uma vez que, com a elevação de níveis de inclusão do coproduto, ocorre a redução da fonte energética (milho) das dietas experimentais (Tabela I). Com o intuito de manter o balanço energético, Ramos et al. (2006) e Zanetti et al. (2014) recomendaram a adição de óleo vegetal às dietas contendo polpa de caju desidratada e resíduo de acerola, respectivamente, para frangos de corte.

O peso absoluto ao abate, os pesos absolutos e relativos de carcaça, cortes nobres, vísceras comestíveis e de gordura abdominal das codornas aos 42 dias de idade não foram $(\mathrm{P}>0,05)$ influenciados pelos diferentes níveis de inclusão do resíduo da polpa de goiaba (Tabela III), evidenciando que a inclusão do resíduo de goiaba não interferiu negativamente sobre o rendimento de carcaça. Provavelmente, a ausência de variação sobre as características de carcaças pode ser um resultado esperado quando há utilização de rações isonutritivas.

Resultados semelhantes foram obtidos por Camelo et al. (2015), que recomendaram a inclusão de até $10 \%$ de resíduo de goiaba em dietas para codornas sem comprometer o desempenho produtivo e o rendimento de carcaça. Lira et al. (2010)), ao estudarem a inclusão do resíduo de goiaba para frangos de corte, também não constataram efeito significativo sobre o rendimento de carcaça. Do mesmo modo, Freitas et al. (2011), quando estudaram a substituição do farelo de soja pelo farelo de coco em rações contendo farelo de castanha do caju para frangos de corte, não observaram efeito significativo para o rendimento de carcaça dessas aves.

Embora não tenha ocorrido efeito $(\mathrm{P}>0,05)$ dos níveis de inclusão do resíduo de goiaba, pode-se observar, em valores absolutos, que as aves alimentadas com 3\% do coproduto apresentaram os maiores pesos absoluto e relativo de carcaças comparados aos pesos daquelas que receberam rações contendo os demais níveis avaliados. Ainda, em valores absolutos, verificou-se que o peso absoluto do peito das codornas aumentou em 1,47\% com a inclusão do resíduo de goiaba.

Os pesos absoluto e relativo do fígado das aves não foram influenciados $(\mathrm{P}>0,05)$ pelos níveis de inclusão 
Tabela III. Valores de peso absoluto $(\mathrm{g})$ e relativo $(\%)$ ao abate, de carcaça, de cortes, de vísceras comestíveis e de gordura abdominal de codornas de corte aos 42 dias alimentados com diferentes níveis de resíduo de goiaba (Values of absolute (g) and relative (\%) weight at slaughter, carcass, cuts, edible offal and abdominal fat of quail at 42 days fed different levels of guava residue).

\begin{tabular}{|c|c|c|c|c|c|c|c|}
\hline \multicolumn{3}{|l|}{ Variáveis } & \multicolumn{4}{|c|}{ Níveis de inclusão NS } & \multirow[b]{2}{*}{ EPN } \\
\hline Peso absoluto (g) & $0 \%$ & $3 \%$ & $6 \%$ & $9 \%$ & $12 \%$ & P-valor & \\
\hline Abate & 259,80 & 269,00 & 255,20 & 257,50 & 260,00 & 0,51 & 2,51 \\
\hline Carcaça & 191,60 & 199,40 & 187,80 & 188,40 & 191,60 & 0,34 & 1,93 \\
\hline Peito & 67,21 & 69,41 & 67,07 & 65,84 & 68,21 & 0,71 & 0,79 \\
\hline Pernas & 45,69 & 46,17 & 44,35 & 43,61 & 44,77 & 0,47 & 0,47 \\
\hline Asas & 16,70 & 16,78 & 16,09 & 16,10 & 16,94 & 0,46 & 0,18 \\
\hline Dorso & 51,74 & 55,41 & 51,12 & 51,48 & 53,18 & 0,58 & 0,90 \\
\hline Fígado & 5,19 & 4,88 & 4,47 & 5,04 & 4,81 & 0,17 & 0,09 \\
\hline Coração & 2,21 & 2,33 & 2,10 & 2,15 & 2,21 & 0,11 & 0,02 \\
\hline Moela & 4,79 & 5,18 & 4,72 & 5,12 & 5,10 & 0,18 & 0,07 \\
\hline G. Abdominal & 1,52 & 2,26 & 2,32 & 2,12 & 2,11 & 0,40 & 0,13 \\
\hline \multicolumn{8}{|l|}{ Peso relativo (\%) } \\
\hline Carcaça & 73,78 & 74,16 & 73,58 & 73,17 & 73,72 & 0,92 & 0,32 \\
\hline Peito & 35,07 & 34,77 & 35,73 & 34,95 & 35,59 & 0,51 & 0,20 \\
\hline Pernas & 23,86 & 23,14 & 23,61 & 23,14 & 23,37 & 0,26 & 0,12 \\
\hline Asa & 8,72 & 8,42 & 8,56 & 8,54 & 8,86 & 0,59 & 0,08 \\
\hline Dorso & 26,98 & 27,84 & 27,21 & 27,36 & 27,73 & 0,96 & 0,39 \\
\hline Fígado & 2,70 & 2,44 & 2,38 & 2,67 & 2,51 & 0,09 & 0,04 \\
\hline Coração & 1,15 & 1,17 & 1,11 & 1,14 & 1,15 & 0,69 & 0,01 \\
\hline Moela & 2,50 & 2,60 & 2,51 & 2,72 & 2,66 & 0,23 & 0,03 \\
\hline G. Abdominal & 0,79 & 1,12 & 1,25 & 1,12 & 1,10 & 0,37 & 0,07 \\
\hline
\end{tabular}

Ns - não significativo (P>0,05\%); CV - Coeficiente de variação; EPM - Erro padrão da média.

do resíduo de polpa de goiaba, entretanto, pode-se constatar em valores absolutos uma redução de 7,32 e de 7,04\%, respectivamente. A redução do tamanho do fígado foi ocasionada, provavelmente, pelo aumento do teor de gordura nas dietas contendo maior nível do resíduo de goiaba, resultando em redução da lipogênese e, consequentemente, menor desenvolvimento do órgão. Do mesmo modo, verifica-se um aumento $6,02 \%$ no peso relativo da moela das aves que, possivelmente, foi atribuído pelo elevado teor de fibras nas dietas de maior nível do resíduo de goiaba, que pode ser justificado, provavelmente, pela maior granulometria e quantidade de fibra das rações, pois o resíduo de goiaba é constituído por sementes, polpa e casca, o que pode ter provocado maiores contrações dos músculos da moela, promovendo, assim, maior massa muscular (González et al., 2007). Resultados semelhantes foram obtidos por Pinto et al. (2014), que observaram redução no peso do fígado e aumento no peso da moela de frangos de corte alimentados com resíduo da acerola. Contrariamente, Lira et al. (2010) e Camelo et al. (2015) não constataram efeito da inclusão de resíduo de goiaba sobre o rendimento de fígado das aves.

Os níveis de inclusão do resíduo de goiaba não influenciaram $(\mathrm{P}>0,05)$ a deposição de gordura abdo- minal, evidenciando que mesmo havendo fracionamento da concentração energética pelo aumento da quantidade de fibra nas dietas referente aos níveis crescentes do resíduo as codornas utilizaram a energia contida nas rações de forma eficiente. Estes resultados corroboram os encontrados por Bastos et al. (2007) e Freitas et al. (2006) ao estudarem os efeitos da inclusão de farelo de coco e farelo do resíduo de caju, respectivamente, em dietas para frangos de corte. Por outro lado, Lira et al. (2009) constataram aumento no teor de gordura abdominal de frangos de corte recebendo rações contendo resíduo de goiaba. Contudo, este estudo demonstra, em valores absolutos, aumento de $28,18 \%$ de gordura abdominal das aves aos 42 dias de idade com a inclusão do resíduo de goiaba. Jensen (1991) relatou que ingredientes com valores de energia metabolizável subestimados incorporados às rações, pode ocasionar aumento na relação energia: proteína da ração, o que gera maiores valores de gordura abdominal. Além disso, esse aumento no teor de gordura abdominal pode ser justificado pela adição de óleo vegetal uma vez que, com a elevação de níveis de inclusão do coproduto, ocorreu a redução do milho, fonte energética, das 
Tabela IV. Análise econômica dos níveis de inclusão do resíduo da polpa de goiaba em relação ao peso vivo das aves aos 42 dias de idade (Economic analysis of inclusion levels of guava pulp residue in relation to live weight of quails at 42 days of age).

\begin{tabular}{|c|c|c|c|c|c|c|c|}
\hline \multicolumn{8}{|c|}{ Variáveis econômicas } \\
\hline Níveis (\%) ${ }^{1}$ & RBM & MBM & MBR & RM & IRR & IBEP & IBER \\
\hline 0,0 & 4,14 & 2,84 & 100,00 & 101,90 & 218,79 & 0,1408 & 100,00 \\
\hline 3,0 & 4,30 & 2,95 & 99,44 & 103,72 & 217,85 & 0,1459 & 103,61 \\
\hline 6,0 & 4,08 & 2,72 & 103,32 & 95,48 & 199,02 & 0,1310 & 93,99 \\
\hline 9,0 & 4,11 & 2,73 & 99,78 & 95,90 & 197,00 & 0,1311 & 93,12 \\
\hline 12,0 & 4,16 & 2,77 & 104,13 & 97,51 & 199,98 & 0,1339 & 95,11 \\
\hline
\end{tabular}

${ }^{1}$ Níveis de inclusão do resíduo de goiaba. RBM = Renda bruta média (R\$/ave); $\mathrm{MBM}=$ Margem bruta média (R\$/ave); $\mathrm{MBR}=\mathrm{Margem}$ bruta relativa (\%); RM = Rentabilidade média (\%); IRR = Índice relativo de rentabilidade em relação ao tratamento 1 (\%); IBEP = índice bioeconômico ponderado e IBER= índice bioeconômico ponderado relativo ao tratamento 1 (\%).

rações experimentais. Lima et al. (1996) afirmam que uma maior quantidade de óleo na dieta em níveis de até 3\% de inclusão causa maior deposição de gordura abdominal em relação a carcaça eviscerada.

Dentro do contexto, pode-se inferir que a composição química do resíduo de polpa de goiaba não influenciou de forma negativa a digestibilidade dos nutrientes e consequentemente o desempenho e o rendimento de carcaça das aves.

O nível de 3,0\% de inclusão do resíduo da polpa de goiaba apresentou superioridade para a renda média bruta $(R \$ 4,30)$, que representa o montante recebido por codorna alimentada, em relação à comercialização das aves alimentadas com os demais níveis (Tabela IV).

Houve superioridade dos níveis 3,0 e 12,0\% de inclusão do resíduo de goiaba para a margem bruta média e a margem bruta relativa, respectivamente. A rentabilidade média e o índice relativo de rentabilidade foram maiores para o nível de 3,0\% do resíduo de goiaba em relação aos demais níveis de inclusão avaliados. O uso do resíduo de goiaba na alimentação de codornas pode ser vantajoso durante períodos de entressafra, quando os preços dos ingredientes tradicionais, como milho e farelo de soja, estão elevados. Dentro desse contexto, a utilização de ingredientes alternativos está diretamente ligada ao preço das matérias-primas, bem como o custo de uma possível suplementação necessária para manter níveis nutricionais adequados para o desempenho das codornas.

\section{CONCLUSÃO}

Recomenda-se a inclusão de $3 \%$ do resíduo de polpa de goiaba na alimentação de codornas de corte, sem que haja comprometimento no desempenho produtivo, rendimento de carcaça e viabilidade econômica, durante o período de um a 42 dias de idade.

\section{BIBLIOGRAFIA}

Anuário brasileiro da fruticultura 2017 / Cleonice de Carvalho ... [et al.]. - Santa Cruz do Sul: Editora Gazeta Santa Cruz,
2017. 88p.: il. ISSN 1808-4931. Disponível em http://www. editoragazeta.com.br/sitewp/wp-content/uploads/2017/03/ PDF-Fruticultura_2017.pdf Acesso em: 23 de março de 2018.

Associação Brasileira de proteína Animal 2018, Relatório Anual 2018: Carne de frangos. Disponível em: http://www.abpa-br. com.br/storage/files/relatorio-anual-2018.pdf Acessado em 02 de junho de 2018.

Bastos, SC, Fuentes MFF, Freitas, ER Espíndola, GB \& Braga, CVP 2007, 'Efeito da inclusão do farelo de coco em rações para frangos de corte', Revista Ciência Agronômica, Fortaleza, v.38, n.3, pp. 297-303.

BRASIL. Ministério da Agricultura, Pecuária e Abastecimento. 'Regulamento de Inspeção Industrial e Sanitária de Produtos de Origem Animal'. Decreto n ${ }^{\circ}$ 30.691, de 29 de março de 1952, e alterações. Diário Oficial da União. Brasília, 1997. Disponível em: www.agricultura.gov.br Acessado em: 29 de dezembro de 2017.

Buffington, DE, Collazo-Arocho, A, Canton, GH, Pitt, D, Thatcher, WW \& Collier, RJ 1981, 'Black globe-humidity index (BGHI) as comfort equation for dairy cows' Transaction of the American Society of Agricultural Engineering, v.24, pp.711-714.

Camelo, LCL, Lana, GRQ, Santos, MJB, Camelo, YARP, Marinho, AL, \& Rabello, CBV 2015, 'Inclusão de farelo de goiaba na dieta de codornas europeias', Ciência Animal. Brasileira, Goiânia, v.16, n.3, pp. 343-349.

Freitas, ER, Fuentes, MFF, Santos Júnior, A, Guerreiro, MEF, \& Espíndola, GB 2006, 'Farelo de castanha de caju em rações para frangos de corte', Pesquisa Agropecuária Brasileira, v.41 , n.6, pp.101-1006.

Freitas, E.R., LIMA, RC, SILVA, RB, Sucupira, FS, Moreira, RF, \& Lopes, IRV 2011 , 'Substituição do farelo de soja pelo farelo de coco em rações contendo farelo da castanha de caju para frangos de corte'. Revista Brasileira de Zootecnia, v.40, n.5, pp.1006-1013.

González-Alvaro, JM, Jiménez-Moreno, E, Lázaro, R., Mateos, GG 2007, 'Effect of type of cereal, heat processing of the cereal, and inclusion of fiber in the diet on performance and digestive traits of broilers', Poultry. Science., v.86, n.8, pp.1705-1715, 2007.

Jansen, WMMA \& Care, B 1989, 'Influence of fiber on digestibility of poultry feeds'. In: Cole, DJA \& Haresign, W, Recent developments in poultry nutrition. London: Butterworths, pp.78-93.

Jensen, LS 1991, 'Subproductos de animales en las formulaciones', Industria Avícola, v.38, pp.28-31.

Lana, GRQ 2000, Avicultura. Recife: UFRPE, 2000. 268 p.

Lima, CAR, Sales, GS, \& Curvello, FA 1996, 'Efeito do uso de óleo em rações de frangos de corte criados no verão'. In: Conferência 
Apinco De Ciência E Tecnologia Avícola, 1996, Curitiba. Anais... Curitiba: FACTA, p.45.

Lira, RC, Rabello, CBV, Silva, EP, Ferreira, PV, Ludke, MCMM, \& Costa, EV 2011, 'Chemical composition and energy value of guava and tomato wastes for broilers chickens at different ages', Revista Brasileira de Zootecnia, v.40, n.5, pp.1019-1024.

Lira, RC, Rabello, CBV, Ferreira, PV, Lana, GRQ, Ludke, JV, \& Dutra Júnior, WM 2009, 'Inclusion of guava wastes in feed for broiler chickens', Revista Brasileira de Zootecnia, v.38, n.12, pp.2401-2407.

Lira, RC, Rabello, CBV, Ludke, MCMM, Ferreira, PV, Lana, GRQ, \& Lana, SRV 2010, 'Productive performance of broiler chickens fed tomato waste', Revista Brasileira de Zootecnia, v.39, n.5, pp. 1074-1081.

Murakami, AE, Moraes, VMB, \& Ariki, J 1993, 'Níveis de proteína e energia em dietas de codornas japonesas (Coturnix Coturnix japonica) em postura', Revista Brasileira de Zootecnia, v.22, p.541-551

Pinto, MF, Moreira, AJC, Bossolani, ILC, Garcia-Neto, M, \& Perri, SHV 2014, 'Production of slow growing broiler chicken using byproduct from fruit juice processing', Archivos Latinoamericanos de Producción Animal, v.22, n.5, pp.32-35.

R Development Core Team: 'A language and environment for statistical computing'. Vienna: R Foundation for Statistical Computing, 2016.

Ramos, LSN, Lopes, JB, Figueiredo, AV, Freitas, AC, Farias, LA, Santos, LS, \& Silva, HO 2006, 'Polpa de caju em rações para frangos de corte na fase final: Desempenho e características de carcaça', Revista Brasileira de Zootecnia. v.35, n.3, pp. 808-810.

Rostagno, HS, Albino, LFT, Donzele, JL, Oliveira, RF, Lopes, DC, Ferreira, AS, Barreto, SLT \& Euclides, RF 2011, Tabelas brasileiras para aves e suínos: composição de alimentos e exigências nutricionais. 3. ed. Viçosa, MG: 2011. 252 p.

Sales, PJP, FURUYA, WM, \& SANTOS, VG 2004, 'Valor nutritivo dos farelos do subproduto industrial do tomate (Lycopersicum esculentum) e da goiaba (Psidium guajava) para tilápia do nilo (Oreochromis niloticus)', In: REUNIÃO ANUAL DA SOCIEDADE BRASILEIRA DE ZOOTECNIA, 41., 2004, Campo Grande. Anais... Campo Grande: SBZ, 2004. (CD-ROM).

Santos, EL Ludke, MCMM, Barbosa, JM, Rabello, CBV, \& Ludke, JV 2009,'Digestibilidade aparente do farelo de coco e do resíduo de goiaba para tilápia do Nilo', Revista Caatinga, v.22, n.2, pp. 175-180.

Silva, DJ, Queiros, A. 2002, Análise de alimentos: métodos químicos e biológicos. 3.ed. Viçosa, MG:UFV, 2002. 235p.

Silva, JDA 1999, 'Composição química e digestibilidade in situ da semente de goiaba (Psidium guajava L.)', Recife, 34 p. Dissertação (Mestrado em Produção Animal) - Universidade Federal Rural de Pernambuco, Recife.

Sousa, MSB, Vieira, LM, Silva, MJM, \& Lima, A 2011 , 'Caracterização nutricional e compostos antioxidantes em resíduos de polpas de frutas tropicais', Ciência e Agrotecnologia, v.35, n.3, pp.554-559. Tardocchi, CFT, Nobre, RTRS, Bonaparte, TP, \& Cabral, NO, 2014, 'Digestibilidade de resíduos agroindustriais para suínos na fase inicial'. Revista Eletrônica Nutritime, v. 1 1, n.06, pp. 3770-3780.

Zanetti, LH, Polycarpo, GV, Brichi, ALC, Barbieri, A, Oliveira, RF, Sabbag, OJ, Cooke, RF, \& Cruz-Polycarpo, VC 2014, 'Performance and economic analysis of broilers fed diets containing acerola meal in replacement of corn'. Brazilian Journal of Veterinary Research and Animal Science, v.51, n.3, pp.224-232.Jensen, LS 1991, 'Subproductos de animales en las formulaciones', Industria Avícola, v.38, pp.28-31

Lana, GRQ 2000, Avicultura. Recife: UFRPE, 2000. 268 p.

Lima, CAR, Sales, GS, \& Curvello, FA 1996, 'Efeito do uso de óleo em rações de frangos de corte criados no verão'. In: Conferência Apinco De Ciência E Tecnologia Avícola, 1996, Curitiba. Anais. Curitiba: FACTA, p.45.

Lira, RC, Rabello, CBV, Silva, EP, Ferreira, PV, Ludke, MCMM, \& Costa, EV 2011, 'Chemical composition and energy value of guava and tomato wastes for broilers chickens at different ages', Revista Brasileira de Zootecnia, v.40, n.5, pp.1019-1024.

Lira, RC, Rabello, CBV, Ferreira, PV, Lana, GRQ, Ludke, JV, \& Dutra Júnior, WM 2009, 'Inclusion of guava wastes in feed for broiler chickens', Revista Brasileira de Zootecnia, v.38, n.12, pp.2401-2407.

Lira, RC, Rabello, CBV, Ludke, MCMM, Ferreira, PV, Lana, GRQ, \& Lana, SRV 2010, 'Productive performance of broiler chickens fed tomato waste', Revista Brasileira de Zootecnia, v.39, n.5, pp.1074-1081.

Murakami, AE, Moraes, VMB, \& Ariki, J 1993, 'Níveis de proteína e energia em dietas de codornas japonesas (Coturnix Coturnix japonica) em postura', Revista Brasileira de Zootecnia, v.22, p.541-551.

Pinto, MF, Moreira, AJC, Bossolani, ILC, Garcia-Neto, M, \& Perri, SHV 2014, 'Production of slow growing broiler chicken using byproduct from fruit juice processing', Archivos Latinoamericanos de Producción Animal, v.22, n.5, pp.32-35.

R Development Core Team: 'A language and environment for statistical computing'. Vienna: R Foundation for Statistical Computing, 2016.

Ramos, LSN, Lopes, JB, Figueiredo, AV, Freitas, AC, Farias, LA, Santos, LS, \& Silva, HO 2006, 'Polpa de caju em rações para frangos de corte na fase final: Desempenho e características de carcaça', Revista Brasileira de Zootecnia. v.35, n.3, pp.808-810.

Rostagno, HS, Albino, LFT, Donzele, JL, Oliveira, RF, Lopes, DC, Ferreira, AS, Barreto, SLT \& Euclides, RF 2011 , Tabelas brasileiras para aves e suínos: composição de alimentos e exigências nutricionais. 3. ed. Viçosa, MG: 2011. 252 p.

Sales, PJP, FURUYA, WM, \& SANTOS, VG 2004, 'Valor nutritivo dos farelos do subproduto industrial do tomate (Lycopersicum esculentum) e da goiaba (Psidium guajava) para tilápia do nilo (Oreochromis niloticus)', In: REUNIÃO ANUAL DA SOCIEDADE BRASILEIRA DE ZOOTECNIA, 41., 2004, Campo Grande. Anais... Campo Grande: SBZ, 2004. (CD-ROM).

Santos, EL Ludke, MCMM, Barbosa, JM, Rabello, CBV, \& Ludke, JV 2009 ,'Digestibilidade aparente do farelo de coco e do resíduo de goiaba para tilápia do Nilo', Revista Caatinga, v.22, n.2, pp.175-180.

Silva, DJ, Queiros, A. 2002, Análise de alimentos: métodos químicos e biológicos. 3.ed. Viçosa, MG:UFV, 2002. 235p. 
Silva, JDA 1999, 'Composição química e digestibilidade in situ da semente de goiaba (Psidium guajava L.)', Recife, 34 p. Dissertação (Mestrado em Produção Animal) - Universidade Federal Rural de Pernambuco, Recife.

Sousa, MSB, Vieira, LM, Silva, MJM, \& Lima, A 2011, 'Caracterização nutricional e compostos antioxidantes em resíduos de polpas de frutas tropicais', Ciência e Agrotecnologia, v.35, n.3, pp.554-559.
Tardocchi, CFT, Nobre, RTRS, Bonaparte, TP, \& Cabral, NO, 2014, 'Digestibilidade de resíduos agroindustriais para suínos na fase inicial'. Revista Eletrônica Nutritime, v. 11 , n.06, pp. 3770-3780.

Zanetti, LH, Polycarpo, GV, Brichi, ALC, Barbieri, A, Oliveira, RF, Sabbag, OJ, Cooke, RF, \& Cruz-Polycarpo, VC 2014, 'Performance and economic analysis of broilers fed diets containing acerola meal in replacement of corn'. Brazilian Journal of Veterinary Research and Animal Science, v.51, n.3, pp.224-232. 